\title{
A THEORY AND CHALLENGES FOR COARSENING IN MICROSTRUCTURE
}

\author{
KATAYUN BARMAK \\ Department of Applied Physics and Applied Mathematics, Columbia University, New York, NY 10027 \\ Eva EgGELING \\ Fraunhofer Austria Research GmbH, Visual Computing, A-8010 Graz, Austria \\ Maria EMELianenKo \\ Department of Mathematical Sciences, George Mason University, Fairfax, VA 22030 \\ YEKATERINA EPSHTEYN \\ Department of Mathematics, The University of Utah, Salt Lake City, UT, 84112 \\ DAVID KINDERLEHRER \\ Department of Mathematical Sciences, Carnegie Mellon University, Pittsburgh, PA 15213 \\ RICHARD SHARP \\ Microsoft Corporation One Microsoft Way Redmond, WA 98052 \\ SHLOMO TA'ASAN \\ Department of Mathematical Sciences, Carnegie Mellon University, Pittsburgh, PA 15213
}

Dedicated to the memory of Enrico Magenes.

1991 Mathematics Subject Classification. Primary: 37M05, 35Q80, 93E03, 60J60, 35K15, 35A15.

Key words and phrases. Coarsening, Texture Development, Large Metastable Networks, Large scale simulation, Critical Event Model, Entropy Based Theory, Free Energy, Fokker-Planck Equation, KantorovichRubinstein-Wasserstein Metric, convex duality.

Research supported by NSF DMR0520425, DMS 0405343, DMS 0305794, DMS 0806703, DMS 0635983, DMS 0915013, DMS 1056821, DMS 1216433, OISE 0967140, DMS 1112984. 
ABstract. Cellular networks are ubiquitous in nature. Most engineered materials are polycrystalline microstructures composed of a myriad of small grains separated by grain boundaries, thus comprising cellular networks. The grain boundary character distribution (GBCD) is an empirical distribution of the relative length (in 2D) or area (in 3D) of interface with a given lattice misorientation and normal. During the coarsening, or growth, process, an initially random grain boundary arrangement reaches a steady state that is strongly correlated to the interfacial energy density. In simulation, if the given energy density depends only on lattice misorientation, then the steady state GBCD and the energy are related by a Boltzmann distribution. This is among the simplest non-random distributions, corresponding to independent trials with respect to the energy. Why does such simplicity emerge from such complexity?

Here we an describe an entropy based theory which suggests that the evolution of the GBCD satisfies a Fokker-Planck Equation, an equation whose stationary state is a Boltzmann distribution. The properties of the evolving network that characterize the GBCD must be identified and appropriately upscaled or 'coarse-grained'. This entails identifying the evolution of the statistic in terms of the recently discovered Monge-KantorovichWasserstein implicit scheme. The undetermined diffusion coefficient or temperature parameter is found by means of a convex optimization problem reminiscent of large deviation theory.

\section{Contents}

1. Introduction 2

2. Reprise of Mesoscale theory 4

3. Discussion of the Simulation 7

4. A Simplified coarsening model with entropy and dissipation 9

4.1. Formulation 10

4.2. The mass transport paradigm 13

5. Validation of the scheme $\quad 15$

$\begin{array}{ll}\text { 5.1. An example of the simplified problem } & 17\end{array}$

6. The entropy method for the GBCD 18

6.1. Quadratic interfacial energy density 18

6.2. Quartic interfacial energy density 18

6.3. Remarks on a Theory for the Diffusion Coefficient $\sigma$ or the Temperature-Like

Parameter 21

7. Closing comments 21

Acknowledgements $\quad 22$

$\begin{array}{ll}\text { References } & 22\end{array}$

\section{INTRODUCTION}

Cellular networks are ubiquitous in nature. They exhibit behavior on many different length and time scales and are generally metastable. Most technologically useful materials are polycrystalline microstructures composed of a myriad of small monocrystalline 
grains separated by grain boundaries, and thus comprise cellular networks. The energetics and connectivity of the grain boundary network plays a crucial role in determining the properties of a material across a wide range of scales. A central problem is to develop technologies capable of producing an arrangement of grains that provides for a desired set of material properties. Traditionally the focus has been on distributions of geometric features, like cell size, and a preferred distribution of grain orientations, termed texture. Attaining these gives the configuration order in a statistical sense. More recent mesoscale experiment and simulation permit harvesting large amounts of information about both geometric features and crystallography of the boundary network in material microstructures, [2],[1],[43],[58],[59]. This has led us to the notion of the Grain Boundary Character Distribution.

The grain boundary character distribution (GBCD) is an empirical distribution of the relative length (in $2 \mathrm{D}$ ) or area (in $3 \mathrm{D}$ ) of interface with a given lattice misorientation and grain boundary normal.

A first discovery is that during the growth process, an initially random grain boundary arrangement reaches a steady state that is strongly correlated to the interfacial energy density. In simulation, a stationary GBCD is always found. Moreover there is consistency between experimental GBCD's and simulated GBCD's. The boundary network of a cellular structure is naturally ordered.

A second discovery is that if the given interfacial energy depends only on lattice misorientation, then the steady state GBCD and the density are related by a Boltzmann distribution. This is among the simplest non-random distributions, corresponding to independent trials with respect to the density. Such straightforward dependence between the character distribution and the interfacial energy offers evidence that the GBCD is a material property. It is a leading candidate to characterize texture of the boundary network [43]. Why does such simplicity emerge from such complexity?

Here we describe our recent work developing an entropy based theory that suggests that the evolving GBCD satisfies a Fokker-Planck Equation, [10],[17], cf. also [11], [9], to which we refer for a more complete exposition. Coarsening in polycrystalline systems is a complicated process involving details of material structure, chemistry, arrangement of grains in the configuration, and environment. In this context, we consider just two competing global features, as articulated by C. S. Smith [60]: cell growth according to a local evolution law and space filling constraints. We shall impose curvature driven growth for the local evolution law, cf. Mullins [54]. Space filling requirements are managed by critical events, rearrangements of the network involving deletion of small contracting cells and facets. The properties of this system that characterize the GBCD must be identified and appropriately upscaled or 'coarse-grained'. For a perspective on these issues, we recommend the article by R. V. Kohn [45].

The general platform for this investigation is large scale computation. Numerical simulations are well established as a major tool in the analysis of many physical systems, see for example [64],[48],[49],[29],[30],[22],[62],[61],[25],[26], [46],[56],[23],[50],[52]. However, the idea of large scale computation as the essential method for the modeling and comprehension 
of large complex systems is relatively new. Porous media and groundwater flow is an important case of this, see for example [33],[5],[4],[7],[6]. For coarsening of cellular systems, it is a natural approach as well. The laboratory is the venue to assess the validity of the local evolution law. Once this law is adopted, we appeal to simulation, since we cannot control all the other elements present in the experimental system, many of which are unknown. On the other hand, in silico we may exercise, or at least we may attempt to exercise, precise control of the variables appropriate to the evolution law and the constraint.

There are many large scale metastable material systems, for example, magnetic hysteresis, [19], and second phase coarsening, [51],[66]. In these, the theory is based on mesoscopic or macroscopic variables simply abstracting the role of the smaller scale elements of the system. There is no general 'multiscale' framework for upscaling from the local behavior of individual cells to behavior of the network when they interact and change their character. So we must attempt to tease the system level information from the many coupled elements of which it consists. This information will be available primarily from the dissipation relation (2.6) which is implied by the balance of forces at triple junctions (2.3), due to Herring, [34],[35]. Lax resolution of the Herring Condition gives rise to an unreliable GBCD.

Our strategy is to introduce a simplified coarsening model that is driven by the boundary conditions and reflects the dissipation relation of the grain growth system. This will be more accessible to analysis. It resembles an ensemble of inertia-free spring-mass-dashpots. For this simpler network, we learn how entropic or diffusive behavior at the large scale emerges from a dissipation relation at the scale of local evolution. The cornerstone is a novel implementation of the iterative scheme for the Fokker-Planck Equation in terms of the system free energy and a Kantorovich-Rubinstein-Wasserstein metric [39], cf. also [38], which will be summarized later in the presentation.

The network level nonequilibrium nature of the scheme leaves undetermined the diffusion constant in the Fokker-Planck Equation, or equivalently the 'temperature parameter' of the Boltzmann Distribution we are seeking. We employ the Kullback-Leibler relative entropy, cf. (5.2), and find a convex duality problem for this parameter. It has a statistical interpretation, or information theory interpretation, in terms of an optimal prefix code, cf. eg. [57], and moreover has evident connections to large deviations. This suggests that had we simply asked to identify an optimal distribution via a known statistical method, we would have been led full circle to entropy methods.

\section{Reprise of Mesoscale theory}

Our point of departure is the common denominator theory for the mesoscale description of microstructure evolution. This is growth by curvature, the Mullins Equation (2.2) below, for the evolution of curves or arcs individually or in a network, which we employ for our local law of evolution. Boundary conditions must be imposed where the arcs meet. This condition is the Herring Condition, (2.3), which is the natural boundary condition at equilibrium for the Mullins Equation. Since their introduction by Mullins, [54], and Herring, [34], [35], a large and distinguished body of work has grown about these equations. Most relevant to here are [32], [20], [42], [55]. Curvature driven growth has old origins, 
dating at least to Burke and Turnbull [21]. Let $\alpha$ denote the misorientation between two grains separated by an $\operatorname{arc} \Gamma$, as noted in Figure 1 , with normal $n=(\cos \theta, \sin \theta)$, tangent direction $b$ and curvature $\kappa$. Let $\psi=\psi(\theta, \alpha)$ denote the energy density on $\Gamma$. So

$$
\Gamma: x=\xi(s, t), \quad 0 \leqq s \leqq L, t>0
$$

with

$$
\begin{aligned}
& b=\frac{\partial \xi}{\partial s} \text { (tangent) and } n=R b \text { (normal) } \\
& v=\frac{\partial \xi}{\partial t} \text { (velocity) and } v_{n}=v \cdot n \text { (normal velocity) }
\end{aligned}
$$

where $R$ is a positive rotation of $\pi / 2$. The Mullins Equation of evolution is

$$
v_{n}=\left(\psi_{\theta \theta}+\psi\right) \kappa \text { on } \Gamma \text {. }
$$

We assume that only triple junctions are stable and that the Herring Condition holds at

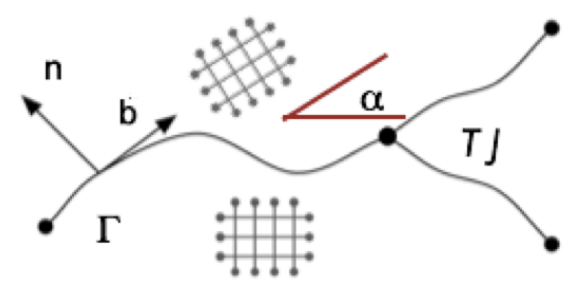

FigURE 1. An $\operatorname{arc} \Gamma$ with normal $n$, tangent $b$, and lattice misorientation $\alpha$, illustrating lattice elements.

triple junctions. This means that whenever three curves, $\left\{\Gamma^{(1)}, \Gamma^{(2)}, \Gamma^{(3)}\right\}$, meet at a point $p$ the force balance, (2.3) below, holds:

$$
\sum_{i=1, . ., 3}\left(\psi_{\theta} n^{(i)}+\psi b^{(i)}\right)=0
$$

It is easy to check that the instantaneous rate of change of energy of $\Gamma$ is

$$
\frac{d}{d t} \int_{\Gamma} \psi|b| d s=-\int_{\Gamma} v_{n}^{2} d s+\left.v \cdot\left(\psi_{\theta} n+\psi b\right)\right|_{\partial \Gamma}
$$

Consider a network of grains bounded by $\left\{\Gamma_{i}\right\}$ subject to some condition at the border of the region they occupy, like fixed end points or periodicity, cf. Figure 2. The important features of the algorithm used in the current simulation are given briefly in the next Section 3. For the description of the previous algorithms the reader can consult [44],[41]. The typical simulation consists in initializing a configuration of cells and their boundary arcs, usually by a modified Voronoi tessellation, and then solving the system (2.2), (2.3), eliminating facets when they have negligible length and cells when they have negligible area, cf. Section 3. The total energy of the system is given by 


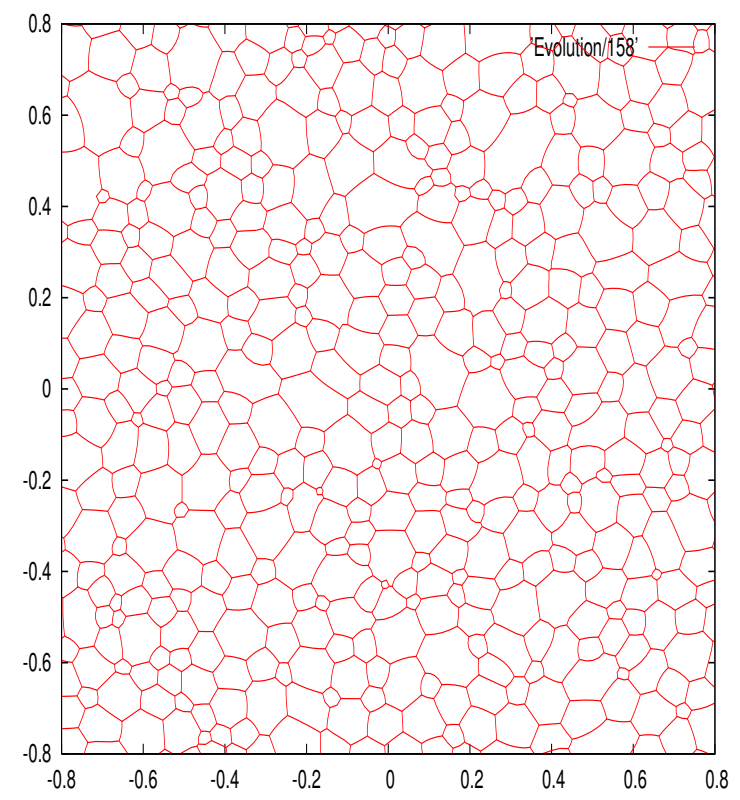

FigurE 2. Example of an instant during the simulated evolution of a cellular network. This is from a small simulation with constant energy density and periodic conditions at the border of the configuration.

$$
E(t)=\sum_{\left\{\Gamma_{i}\right\}} \int_{\Gamma_{i}} \psi|b| d s
$$

Owing exactly to the Herring Condition (2.3), the instantaneous rate of change of the energy

$$
\begin{aligned}
\frac{d}{d t} E(t) & =-\sum_{\left\{\Gamma_{i}\right\}} \int_{\Gamma_{i}} v_{n}^{2} d s+\sum_{T J} v \cdot \sum\left(\psi_{\theta} n+\psi b\right) \\
& =-\sum_{\left\{\Gamma_{i}\right\}} \int_{\Gamma_{i}} v_{n}^{2} d s \\
& \leqq 0
\end{aligned}
$$

rendering the network dissipative for the energy in any instant absent of critical events. Indeed, in an interval $\left(t_{0}, t_{0}+\tau\right)$ where there are no critical events, we may integrate $(2.6)$ to obtain a local dissipation equation

$$
\sum_{\left\{\Gamma_{i}\right\}} \int_{t_{0}}^{t_{0}+\tau} \int_{\Gamma_{i}} v_{n}^{2} d s d t+E\left(t_{0}+\tau\right)=E\left(t_{0}\right)
$$


which bears a strong resemblance to the simple dissipation relation for an ensemble of inertia free springs with friction. In the simulation, the facet interchange and cell deletion are arranged so that (2.6) is maintained.

Suppose, for simplicity, that the energy density is independent of the normal direction, so $\psi=\psi(\alpha)$. It is this situation that will concern us here. Then (2.2) and (2.3) may be expressed

$$
\begin{aligned}
v_{n} & =\psi \kappa \text { on } \Gamma \\
\sum_{i=1, \ldots, 3} \psi b^{(i)} & =0 \text { at } p
\end{aligned}
$$

where $p$ denotes a triple junction. (2.9) is the same as the Young wetting law.

For this situation we define the grain boundary character distribution, GBCD,

$$
\begin{aligned}
& \rho(\alpha, t)=\text { relative length of arc of misorientation } \alpha \text { at time } t, \\
& \text { normalized so that } \int_{\Omega} \rho d \alpha=1
\end{aligned}
$$

\section{Discussion of the Simulation}

Our simulation method involves a boundary tracking approach which is in contrast to level set methods, for example recent work [24], and phase field methods, for example $[28],[40]$, used by other groups. We approximate only the network of grain boundaries while other methods involve the interior of the grains as well. The location of grain boundaries is implicit in these methods. The advantage of our method is the flexibility in applying a selected energy density on curves and the boundary conditions at triple junctions. The evolution in our approach is based on a variational approach for solving numerically the system (2.2), (2.3) for the network while managing the critical events. It must be designed so it is robust and reliable statistics can be harvested. Owing to the size and complexity of the network there are number of challenges in the designing of the method. These include

- management of the data structure of cells, facets, and triple junctions, dynamic because of critical events,

- management of the computational domain

- initialization of the computation,

- maintaining the triple junction boundary condition (2.3) while

- resolving the equations (2.2) with sufficient accuracy

We will address some of these issues below. We also need some diagnostics to understand the accuracy of the physical model and of the numerical scheme. Questions of numerical accuracy can be addressed by mesh refinement and the convergence of the approximate solutions. For physical accuracy of the model we look at certain properties of the solutions. For example, it is known that the average area of cells grows linearly even in very casual simulations of coarsening, although more careful diagnostics show that the Herring Condition (2.3) in these efforts fails. As noted in the introduction, this will lead to an unreliable 
determination of the GBCD.

In view of the dissipation inequality (2.6) the evolution of the grain boundary system may be viewed as a modified steepest descent for the energy. Therefore, the cornerstone of our scheme which assures its stability is the discrete dissipation inequality for the total grain boundary energy which holds when the discrete Herring Condition is satisfied. In general, discrete dissipation principles ensure the stability and convergence of numerical schemes to the continuous solution. The design of our numerical scheme is based on a weak formulation, a variational principle which avoids the additional complexity of higher order spaces. In particular, there is no explicit use of curvature which is the case for direct discretization of equations (2.2), (2.3).

The simulation of the grain network is done in three steps by evolving first the grain boundaries, according to Mullin's equation (2.2), and then updating the triple points according to Herring's boundary condition (2.3), imposed at the triple junctions, and finally managing the rearrangement events. In our numerical simulations, grain boundaries are defined by the set of nodal points and are approximated using linear elements. In the algorithm, we define a global mesh size, $h$, and uniformly discretized grain boundaries with local mesh size (distance between neighboring nodal points) which depends on $h$. Due to the frequency of critical events, we have used a first order method in time, namely the Forward Euler method. Increasing the order of time discretization to 2 by using a predictor corrector method did not affect the distribution functions, which is the focus of this study. Resolution of the Herring Condition: To satisfy the Herring Condition (2.3) one has to solve the nonlinear equation to determine the new position of the triple junction [44]. We use the Newton method with line search [37] to approximate the new position for the triple junction. As the initial guess for Newton's method, we determine the position of the triple point by defining the velocity of the triple junction to be proportional to the total line stress at that point with coefficient of the proportionality equal to the mobility. This is also dissipative for the network. The Newton algorithm stops if it exceeds a certain tolerance on the number of the iterations. If the Newton algorithm converges, the Herring Condition (2.3) is satisfied to machine precision accuracy at the new position of the triple junction. If the Newton algorithm fails to converge at some triple junctions (this happens when we work with very small cells) we use our initial guess to update the triple junction position.

Critical events: As grain growth proceeds, critical events occur. When grain boundaries (GB) shrink below a certain size, they trigger one or more of the following processes (i) short GB removal, (ii) splitting of unstable junctions (where more then three GB meet) (ii) fixing double GB (GB that share two vertices).

Removal of short GB: A short GB whose length is decreasing is removed. If its length is increasing, it is not removed.

Splitting unstable vertices: When a GB disappears, new vertices may appear where more than three edges meet. These are unstable junctions which are split by introducing a new vertex and a new GB of short length. This step reduces the number of edges meeting at the 
unstable junctions. This process continues until all vertices are triple junctions. Details of each split are designed to maximally decrease the energy.

\section{A Simplified COARSEning Model With Entropy AND DisSipation}

The coarsening process is irreversible because of its dissipative nature. Even in an interlude when there are no rearrangement events, (2.7) shows that a configuration cannot evolve to a former state from a later one. This could be a source of entropy for the system. In our investigation, we view the principal source of entropy to be configurational since we observe the evolution of an 'upscaled' ensemble represented by a single statistic, the misorientation $\alpha$, neglecting the remaining information. This is also a source of irreversibility since we have forgotten information. We return to this shortly.

A significant difficulty in developing a theory for the GBCD, and understanding texture development in general, lies in the lack of understanding of consequences of rearrangement events or critical events, facet interchange and grain deletion, on network level properties. For example, in Fig. 3, the average area of five-faceted grains during a growth experiment on an $A l$ thin film and the average area of five-faceted cells in a typical simulation both increase with time. Now the von Neumann-Mullins Rule is that the area $A_{n}$ of a cell with n-facets satisfies

$$
A_{n}^{\prime}(t)=c(n-6)
$$

when $\psi=$ const. and triple junctions meet at angles of $2 \pi / 3,[53],[65]$. This is thought to hold approximately when anisotropy is small. The von Neumann-Mullins Rule does not fail in the example above, of course, but cells observed at later times had $6,7,8, \ldots$ facets at earlier times. Thus in the network setting, changes which rearrange the network play a major role.

To address these issues, we will examine a much simpler $1 \mathrm{D}$ model which retains kinetics and critical events but neglects curvature driven growth of the boundaries. In our view, there are two important features of the coarsening system: the evolution of the network by steepest descent of the surface energy and the irreversible change/disappearance of the grain boundaries at certain discrete times, which is necessary because the entire configuration is confined. Elaborating on the latter in the two-dimensional setting of Fig.1: at most times the evolution is smooth, but once in a while a pair of neighboring triple points collides and the grain boundary that joins them disappears forever.

We have used this model to develop a statistical theory for critical events, [15],[16],[14]. It has been found to have its own GBCD as well, $[9],[11],[10],[17]$, which we shall now review.

Our main idea in [9],[11],[10],[17] is that the GBCD statistic for the simplified model resembles the solution of a Fokker-Planck Equation via the mass transport implicit scheme, [39]. In [9],,[11],[10],[17] the simplified model is formulated as a gradient flow which results in a dissipation inequality analogous to the one found for the coarsening grain network. Because of this simplicity, it will be possible to 'upscale' the network level system description to a higher level GBCD description that accomodates irreversibility. A more useful 

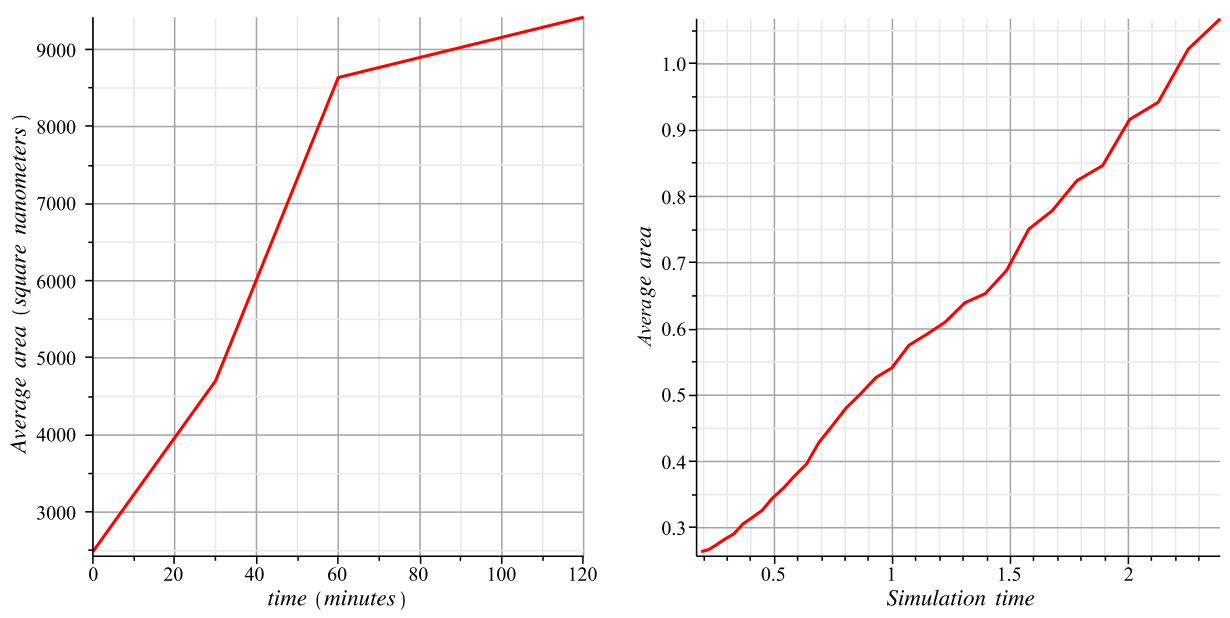

FIGURE 3. The average area of five-sided cell populations during coarsening in two different cellular systems showing that the von Neumann-Mullins $n-6$-Rule (4.1) does not hold at the scale of the network. (left) In an experiment on $A l$ thin film, [8], and (right) a typical simulation (arbitrary units).

dissipation inequality is obtained by modifying the viscous term to be a mass transport term, which now brings us to the realm of the Kantorovich-Rubinstein-Wasserstein implicit scheme. As this changes the ensemble, there is an entropic contribution, which we take to be proportional to configurational entropy. This then suggests the Fokker-Planck paradigm.

However, we do not know that the statistic solves the Fokker-Planck PDE but we can ask if it shares important aspects of Fokker-Planck behavior. We give evidence for this by asking for the unique 'temperature-like' parameter, the factor noted above, the relative entropy achieves a minimum over long time. The empirical stationary distribution and Boltzmann distribution with the special value of 'temperature' are in excellent agreement. This gives an explanation for the stationary distribution and the kinetics of evolution. At this point of our investigations, we do not know that the two dimensional network has the detailed dissipative structure of the simplified model, but we are able to produce evidence that the same argument employing the relative entropy does suggest the correct kinetics and stationary distribution.

4.1. Formulation. The simplified coarsening model, driven by the boundary conditions, reflects the dissipation relation of the grain growth system. It resembles an ensemble of inertia-free spring-mass-dashpots. It is an abstraction of the role of triple junctions in the presence of the rearrangement events.

Let $I \subset \mathbf{R}$ be an interval of length $L$ partitioned by points $x_{i}, i=1, \ldots, n$, where $x_{i}<x_{i+1}, i=1, \ldots, n-1$ and $x_{n+1}$ identified with $x_{1}$. For each interval $\left[x_{i}, x_{i+1}\right], i=$ $1, \ldots, n$ select a random misorientation number $\alpha_{i} \in(-\pi / 4, \pi / 4]$. The intervals $\left[x_{i}, x_{i+1}\right]$ correspond to grain boundaries (but not the 1D "grain") with misorientations $\alpha_{i}$ and the 
points $x_{i}$ represent the triple junctions. Choose an energy density $\psi(\alpha) \geqq 0$ and introduce the energy

$$
E=\sum_{i=1, \ldots, n} \psi\left(\alpha_{i}\right)\left(x_{i+1}-x_{i}\right)
$$

To have consistency with the evolution of the $2 \mathrm{D}$ cellular network, we impose gradient flow kinetics with respect to (4.2), which is just the system of ordinary differential equations

$$
\begin{aligned}
& \frac{d x_{i}}{d t}=-\frac{\partial E}{\partial x_{i}}, i=1, \ldots, n, \text { that is } \\
& \frac{d x_{i}}{d t}=\psi\left(\alpha_{i}\right)-\psi\left(\alpha_{i-1}\right), i=2 \ldots n, \text { and } \frac{d x_{1}}{d t}=\psi\left(\alpha_{1}\right)-\psi\left(\alpha_{n}\right) .
\end{aligned}
$$

The velocity $v_{i}$ of the $i^{\text {th }}$ boundary is

$$
v_{i}=\frac{d x_{i+1}}{d t}-\frac{d x_{i}}{d t}=\psi\left(\alpha_{i-1}\right)-2 \psi\left(\alpha_{i}\right)+\psi\left(\alpha_{i+1}\right) .
$$

The grain boundary velocities are constant until one of the boundaries collapses. That segment is removed from the list of current grain boundaries and the velocities of its two neighbors are changed due to the emergence of a new junction. Each such deletion event rearranges the network and, therefore, affects its subsequent evolution just as in the two dimensional cellular network. Actually, since the interval velocities are constant, this gradient flow is just a sorting problem. At any time, the next deletion event occurs at smallest positive value of

$$
\frac{x_{i}-x_{i+1}}{v_{i}} .
$$

The length $l_{i}(t)$ of the $i^{\text {th }}$ interval is linear in $t$ until it reaches 0 or until a collision event, when it becomes linear with a different slope. In any event, it is continuous, so $E(t), t>0$, the sum of such functions multiplied by factors, is continuous.

At any time $t$ between deletion events,

$$
\frac{d E}{d t}==-\sum \frac{d x_{i}^{2}}{d t} \leqq 0 .
$$

Next consider for the 1D system (4.3), a time interval $\left(t_{0}, t_{0}+\tau\right)$ with no critical events for now. Then we obtain a grain growth analog of the spring-mass-dashpot-like local dissipation inequality.

$$
\sum_{i=1 \ldots n} \int_{0}^{\tau} \frac{d x_{i}^{2}}{d t} d t+E\left(t_{0}+\tau\right)=E\left(t_{0}\right)
$$

With an appropriate interpretation of the sum, (4.6) holds for all $t_{0}$ and almost every $\tau$ sufficiently small. The dissipation equality (4.6) can also be rewritten in terms of grain boundary velocities as follows:

$$
\frac{1}{4} \sum_{i=1 \ldots n} \int_{0}^{\tau} v_{i}^{2} d t+E\left(t_{0}+\tau\right) \leqq E\left(t_{0}\right)
$$


The energy of the system at time $t_{0}+\tau$ is determined by its state at time $t_{0}$. Vice versa, changing the sign on the right hand side of (4.3) allows us to begin with the state at time $t_{0}+\tau$ and return to the state of time $t_{0}$ : the system is reversible in an interval of time absent of rearragement events. This is no longer the situation after such an event. At the later time, we have no knowledge about which interval, now no longer in the inventory, was deleted.

As explained in [11],[10],[17], we can introduce now the idea of GBCD for the simplified 1D model. Let us consider a new ensemble based on the misorientation parameter $\alpha$ where we take $\Omega:-\frac{\pi}{4} \leqq \alpha \leqq \frac{\pi}{4}$, for later ease of comparison with the two dimensional network for which we are imposing "cubic" symmetry, i.e., "square" symmetry in the plane. The $G B C D$ or character distribution in this context is, as expected, the histogram of lengths of intervals sorted by misorientation $\alpha$ scaled to be a probability distribution on $\Omega$. To be precise, we let

$$
\begin{aligned}
l_{i}(\alpha, t)= & x_{i+1}(t)-x_{i}(t) \\
= & \text { length of the } i^{t h} \text { interval, where explicit note has been taken of } \\
& \text { its misorientation parameter } \alpha
\end{aligned}
$$

Partition $\Omega$ into $m$ subintervals of length $h=\frac{\pi}{2} \frac{1}{m}$ and define

$$
\rho(\alpha, t):=\sum_{\alpha^{\prime} \in((k-1) h, k h]} l_{i}\left(\alpha^{\prime}, t\right) \cdot \frac{1}{L h}, \text { for }(k-1) h<\alpha \leqq k h .
$$

For this definition of the statistic,

$$
\int_{\Omega} \rho(\alpha, t) d \alpha=1
$$

One may express (4.7) in terms of the character distribution (4.8), which amounts to

$$
\mu_{0} \int_{t_{0}}^{t_{0}+\tau} \int_{\Omega}\left|\frac{\partial \rho}{\partial t}(\alpha, t)\right|^{2} d \alpha d t+\int_{\Omega} \psi(\alpha) \rho\left(\alpha, t_{0}+\tau\right) d \alpha \leqq \int_{\Omega} \psi(\alpha) \rho\left(\alpha, t_{0}\right) d \alpha,
$$

where $\mu_{0}>0$ is some constant.

The expression (4.9) is in terms of the new misorientation level ensemble, upscaled from the local level of the original system. We now introduce, as discussed earlier, the modeling assumption, consistent with the lack of reversibility when rearrangement/or critical events occur and add an entropic contribution to (4.9). We consider a standard configurational entropy,

$$
+\int_{\Omega} \rho \log \rho d \alpha
$$

although this is not the only choice. Minimizing (4.10) favors the uniform state, which would be the situation were $\psi(\alpha)=$ constant. A tantalizing clue to the development of texture will be whether or not this entropy strays from its minimum during the simulation. 
Given that (4.9) holds, we assume now that there is some $\lambda>0$ such that for any $t_{0}$ and $\tau$ sufficiently small that

$$
\mu_{0} \int_{t_{0}}^{t_{0}+\tau} \int_{\Omega}\left(\frac{\partial \rho}{\partial t}\right)^{2} d \alpha d t+\left.\int_{\Omega}(\psi \rho+\lambda \rho \log \rho) d \alpha\right|_{t_{0}+\tau} \leqq\left.\int_{\Omega}(\psi \rho+\lambda \rho \log \rho) d \alpha\right|_{t_{0}}
$$

$E(t)$ was analogous to an internal energy or the energy of a microcanonical ensemble and now

$$
F(\rho)=F_{\lambda}(\rho)=E(t)+\lambda \int_{\Omega} \rho \log \rho d \alpha
$$

is a free energy. The value of the parameter $\lambda$ is unknown and will be determined in the Validation Section 5

4.2. The mass transport paradigm. The kinetics of the simplified problem will be understood by interpreting the dissipation principle for the GBCD in terms of a mass transport implicit scheme. In fact, (4.11) fails as a proper dissipation principle because the first term

$$
\mu_{0} \int_{t_{0}}^{t_{0}+\tau} \int_{\Omega}\left(\frac{\partial \rho}{\partial t}\right)^{2} d \alpha d t
$$

does not represent lost energy due to frictional or viscous forces. For a deformation path $f(\alpha, t), 0 \leqq t \leqq \tau$, of probability densities, this quantity is

$$
\int_{0}^{\tau} \int_{\Omega} v^{2} f d \alpha d t
$$

where $f, v$ are related by the continuity equation and initial and terminal conditions

$$
\begin{aligned}
& f_{t}+(v f)_{\alpha}=0 \text { in } \Omega \times(0, \tau), \text { and } \\
& f(\alpha, 0)=\rho(\alpha, 0), f(\alpha, \tau)=\rho(\alpha, \tau),
\end{aligned}
$$

by analogy with fluids [47], p.53 et seq., and elementary mechanics. (We have set $t_{0}=0$ for convenience.)

On the other hand, by a result of Benamou and Brenier [18], given two probability densities $f^{*}, f$ on $\Omega$, the Wasserstein distance $d\left(f, f^{*}\right)$ between them is given by

$$
\begin{aligned}
& \frac{1}{\tau} d\left(f, f^{*}\right)^{2}=\inf \int_{0}^{\tau} \int_{\Omega} v^{2} f d \xi d t \\
& \text { over deformation paths } f(\xi, t) \text { subject to } \\
& f_{t}+(v f)_{\xi}=0, \quad(\text { continuity equation) } \\
& f(\xi, 0)=f^{*}(\xi), f(\xi, \tau)=f(\xi) \text { (initial and terminal conditions) }
\end{aligned}
$$

Let us briefly review the notion of Kantorovich-Rubinstein-Wasserstein metric, or simply Wasserstein metric. The reader can consult [63], [3] for more detailed exposition of the subject. 
Let $D \subset \mathbf{R}$ be an interval, perhaps infinite, and $f^{*}, f$ a pair of probability densities on $D$ (with finite variance). The quadratic Wasserstein metric or 2-Wasserstein metric is defined to be

$$
\begin{aligned}
& d\left(f, f^{*}\right)^{2}=\inf _{P} \int_{D \times D}|x-y|^{2} d p(x, y) \\
& P=\text { joint distributions for } f, f^{*} \text { on } \bar{D} \times \bar{D},
\end{aligned}
$$

i.e., the marginals of any $p \in P$ are $f, f^{*}$. The metric induces the weak-* topology on $C(\bar{D})^{\prime}$. If $f, f^{*}$ are strictly positive, there is a transfer map which realizes $p$, essentially the solution of the Monge-Kantorovich mass transfer problem for this situation. This means that there is a strictly increasing

$$
\begin{aligned}
& \phi: D \rightarrow D \text { such that } \\
& \int_{D} \zeta(y) f(y) d y=\int_{D} \zeta(\phi(x)) f^{*}(x) d x, \zeta \in C(\bar{D}), \text { and } \\
& d\left(f, f^{*}\right)^{2}=\int_{D}|x-\phi(x)|^{2} f^{*} d x
\end{aligned}
$$

In this one dimensional situation, as was known to Frechét, [27],

$$
\begin{aligned}
& \phi(x)=F^{*-1}(F(x)), x \in D, \text { where } \\
& F^{*}(x)=\int_{-\infty}^{x} f^{*}\left(x^{\prime}\right) d x^{\prime} \text { and } F(x)=\int_{-\infty}^{x} f\left(x^{\prime}\right) d x^{\prime}
\end{aligned}
$$

are the distribution functions of $f^{*}, f$. In one dimension there is only one transfer map. The conditions (4.16) are in 'Eulerian' form. Likewise there is the 'Lagrangian' form which follows by rewriting (4.16) using the transfer function formulation in (4.18),

$$
\begin{aligned}
& \frac{1}{\tau} d\left(f, f^{*}\right)^{2}=\inf \int_{0}^{\tau} \int_{D} \phi_{t}^{2} f^{*} d x \\
& \text { over transfer paths } \phi(x, t) \text { from } D \text { to } D \text { with } \\
& \phi(x, 0)=x \text { and } \phi(x, \tau)=\phi(x)
\end{aligned}
$$

Therefore, our goal is to replace (4.13) with (4.14). Since the associated metrics induce different topologies, an estimate must involve additional terms. Assume that our statistic $\rho(\alpha, t)$ satisfies

$$
\rho(\alpha, t) \geqq \delta>0 \text { in } \Omega, t>0 .
$$

This is a necessary assumption for our estimates below. In fact, to proceed with the implicit scheme introduced later, it is sufficient to require (4.21) just for the initial data $\rho_{0}(\alpha)$ since this property is inherited by the iterates. We now use the representation (4.16) and we use the deformation path given by $\rho$ itself to calculate that for some $c_{\Omega}>0$,

$$
\begin{aligned}
& \frac{1}{\tau} d\left(\rho, \rho^{*}\right)^{2} \leqq \int_{0}^{\tau} \int_{\Omega} v^{2} \rho d x d t \leqq \frac{c_{\Omega}}{\min _{\Omega} \rho} \int_{0}^{\tau} \int_{\Omega} \frac{\partial \rho}{\partial t}(x, t)^{2} d x d t, \\
& \rho^{*}(x)=\rho(x, 0) \text { and } \rho(x)=\rho(x, \tau),
\end{aligned}
$$

where 0 represents an arbitrary starting time and $\tau$ a relaxation time. 
Thus there is a $\mu>0$ such that for any relaxation time $\tau>0$,

$$
\frac{\mu}{2} \int_{0}^{\tau} \int_{\Omega} v^{2} \rho d \alpha d t+F_{\lambda}(\rho) \leqq F_{\lambda}\left(\rho^{*}\right)
$$

We next replace (4.23) by a minimum principle, arguing that the path given by $\rho(\alpha, t)$ is the one most likely to occur and the minimizing path has the highest probability. For this step, let $\rho^{*}=\rho\left(\cdot, t_{0}\right)$ and $\rho=\rho(\cdot, t+\tau)$. Observe that from (4.16),

$$
\begin{aligned}
& \frac{1}{\tau} d\left(\rho, \rho^{*}\right)^{2}=\inf \int_{0}^{\tau} \int_{\Omega} v^{2} f d \alpha d t \\
& \text { over deformation paths } f(\alpha, t) \text { subject to } \\
& f_{t}+(v f)_{\alpha}=0, \quad(\text { continuity equation) } \\
& f(\xi, 0)=\rho^{*}(\alpha), f(\alpha, \tau)=\rho(\alpha, \tau) \text { (initial and terminal conditions) }
\end{aligned}
$$

where $d$ is the Wasserstein metric. So we may express the minimum principle in the form

$$
\frac{\mu}{2 \tau} d\left(\rho, \rho^{*}\right)^{2}+F_{\lambda}(\rho)=\inf _{\{\eta\}}\left\{\frac{\mu}{2 \tau} d\left(\eta, \rho^{*}\right)^{2}+F_{\lambda}(\eta)\right\}
$$

For each relaxation time $\tau>0$ we determine iteratively the sequence $\left\{\rho^{(k)}\right\}$ by choosing $\rho^{*}=\rho^{(k-1)}$ and $\rho^{(k)}=\rho$ in (4.25) and set

$$
\rho^{(\tau)}(\alpha, t)=\rho^{(k)}(\alpha) \text { in } \Omega \text { for } k \tau \leqq t<(k+1) \tau .
$$

We then anticipate recovering the GBCD $\rho$ as

$$
\rho(\alpha, t)=\lim _{\tau \rightarrow 0} \rho^{(\tau)}(\alpha, t),
$$

with the limit taken in a suitable sense. It is known that $\rho$ obtained from (4.27) is the solution of the Fokker-Planck Equation, [39],

$$
\mu \frac{\partial \rho}{\partial t}=\frac{\partial}{\partial \alpha}\left(\lambda \frac{\partial \rho}{\partial \alpha}+\psi^{\prime} \rho\right) \text { in } \Omega, 0<t<\infty .
$$

We might point out here, as well, that a solution of (4.28) with periodic boundary conditions and nonnegative initial data is positive for $t>0$.

\section{VAlidation OF THE SCHEME}

We now begin the validation step of our model. The procedure which leads to the implicit scheme, based on the dissipation inequality (4.7), holds for the entire system but does not identify individual intermediate 'spring-mass-dashpots'. The consequence is that we cannot set the temperature-like parameter $\sigma$, but in some way must decide if one exists. Introduce the notation for the Boltzmann distribution with parameter $\lambda$

$$
\rho_{\lambda}(\alpha)=\frac{1}{Z_{\lambda}} e^{-\frac{1}{\lambda} \psi(\alpha)}, \alpha \in \Omega, \text { with } Z_{\lambda}=\int_{\Omega} e^{-\frac{1}{\lambda} \psi(\alpha)} d \alpha
$$

With validation we would gain qualitative properties of solutions of (4.28): 
- $\rho(\alpha, t) \rightarrow \rho_{\sigma}(\alpha)$ as $t \rightarrow \infty$, and

- this convergence is exponentially fast.

The Kullback-Leibler relative entropy for (4.28) is given by

$$
\begin{aligned}
& \Phi_{\lambda}(\eta)=\Phi\left(\eta \| \rho_{\lambda}\right)=\int_{\Omega} \eta \log \frac{\eta}{\rho_{\lambda}} d \alpha \text { where } \\
& \eta \geqq 0 \text { in } \Omega, \quad \int_{\Omega} \eta d \alpha=1
\end{aligned}
$$

with $\rho_{\lambda}$ from (5.1). By Jensen's Inequality it is always nonnegative. In terms of the free energy (4.12) and (5.1), (5.2) is given by

$$
\Phi_{\lambda}(\eta)=\frac{1}{\lambda} F_{\lambda}(\eta)+\log Z_{\lambda} .
$$

(Note: In our earlier work $[10,17]$, we defined relative entropy to be $\lambda$ times (5.2).) A solution $\rho$ of (4.28) has the property that

$$
\Phi_{\lambda}(\rho) \rightarrow 0 \text { as } t \rightarrow \infty .
$$

Therefore, we seek to identify the particular $\lambda=\sigma$ for which $\Phi_{\sigma}$ defined by the GBCD statistic $\rho$ tends monotonically to the minimum of all the $\left\{\Phi_{\lambda}\right\}$ as $t$ becomes large. We then ask if the terminal, or equilibrium, empirical distribution $\rho$ is equal to $\rho_{\sigma}$. Note that since

$$
f(x, y)=x \log x-x \log y, x, y>0,
$$

is convex, $\Phi\left(\eta \| \rho_{\lambda}\right)$ is a convex function of $\left(\eta, \rho_{\lambda}\right)$. We assign a time $t=T_{\infty}$ and seek to minimize (5.2) at $T_{\infty}$. With

$$
\psi_{\lambda}=\frac{\psi}{\lambda}+\log Z_{\lambda}
$$

this minimization is a convex duality type of optimization problem, namely, to find the $\sigma$ for which

$$
\int_{\Omega}\left\{\psi_{\sigma} \rho+\rho \log \rho\right\} d \alpha=\inf _{\left\{\psi_{\lambda}\right\}} \int_{\Omega}\left\{\psi_{\lambda} \rho+\rho \log \rho\right\} d \alpha
$$

Note that

$$
\int_{\Omega} e^{-\psi_{\lambda}} d \alpha=1
$$

which gives the minimization in (5.6) the form of finding an optimal prefix code, eg. [57]. Here the potential $\psi_{\lambda}$, the code, is minimized in a family rather than the unknown density $\rho$ itself, which is the given alphabet. For practical purposes, note that

$$
\Phi\left(\rho \| \rho_{\lambda}\right)=\int_{\Omega} \rho \log \rho d \alpha+\frac{1}{\lambda} \int_{\Omega} \psi \rho d \alpha+\log Z_{\lambda}
$$

is a strictly convex non-negative function of the 'inverse temperature' $\beta=\frac{1}{\lambda}, \beta>0$, and thus admits a unique minimum.

The information theory interpretation is that we are minimizing the information loss among trial encodings of the alphabet represented by the statistic $\rho$. In this sense we see 
that asking for an optimal distribution $\rho_{\sigma}$ to represent our statistic $\rho$, necessarily introduces (relative) entropy in our considerations, returning us, as it were, full circle.

From a given simulation, we harvest the GBCD statistic. It is a trial. The convexity of $\Phi\left(\rho \| \rho_{\lambda}\right)$ suggests that we can average trials. For trials $\left\{\rho_{1}, \ldots \rho_{N}\right\}$,

$$
\Phi\left(\frac{1}{N} \sum_{i=1 \ldots N} \rho_{i} \| \rho_{\lambda}\right) \leqq \frac{1}{N} \sum_{i=1 \ldots N} \Phi\left(\rho_{i} \| \rho_{\lambda}\right) .
$$

So we can seek the optimal $\lambda=\sigma$ by optimizing with the averaged trial. We shall illustrate this for the validation process for the two dimensional simulation.

5.1. An example of the simplified problem. For the simplified coarsening model, we consider

$$
\psi(\alpha)=1+2 \alpha^{2} \text { in } \Omega=\left(-\frac{\pi}{4}, \frac{\pi}{4}\right),
$$

and shall identify a unique such parameter, which we label $\sigma$, by seeking the minimum of the relative entropy (5.2), namely by inspection of plots of (5.6) and (5.7), and then comparing $\rho$ with the found $\rho_{\sigma}$. This $\psi$ the development to second order of $\psi(\alpha)=1+0.5 \sin ^{2} 2 \alpha$ used in the $2 \mathrm{D}$ simulation. Moreover, since the potential is quadratic, it represents a version of the Ornstein-Uhlenbeck process. We agree that $T_{\infty}=T(80 \%)=6.73$ represents time equals infinity. This is the time at which $80 \%$ of the segments have been deleted and corresponds to the stationary configuration in the two-dimensional simulation. For the simplified critical event model we are considering, it is clear that by computing for a sufficiently long time, all cells will be gone. This time may be quite long. For comparison, $T(90 \%)=30$ and $T(95 \%)=103$. There may be additional criteria for choosing a $T$ in the neighborhood of $T(80 \%)$ and we may wish to discuss this later. The results are reported in Fig. 4.
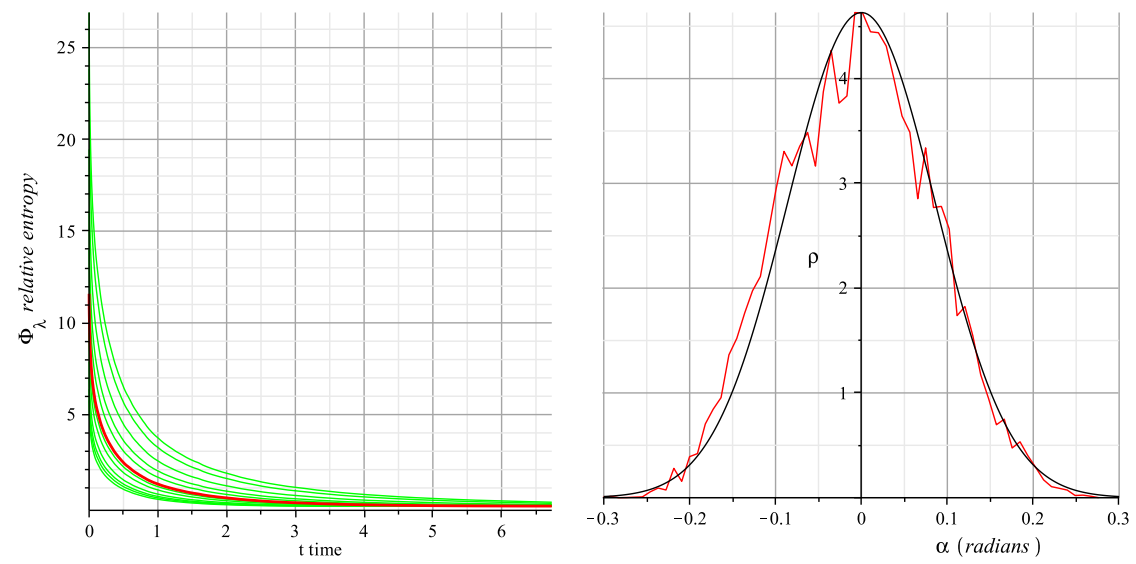

FIGURE 4. Graphical results for the simplified coarsening model. (left) Relative entropy plots for selected values of $\lambda$ with $\Phi_{\sigma}$ noted in red. The value of $\sigma=0.0296915$. (right) Empirical distribution at time $T=T_{\infty}$ in red compared with $\rho_{\sigma}$ in black. 


\section{The ENTRopy Method FOR The GBCD}

6.1. Quadratic interfacial energy density. We shall apply the method of Section 5 to the GBCD harvested from the 2D simulation. We consider first a typical simulation with the energy density

$$
\psi(\alpha)=1+\epsilon(\sin 2 \alpha)^{2},-\frac{\pi}{4} \leqq \alpha \leqq \frac{\pi}{4}, \epsilon=1 / 2,
$$

Figure 5, initialized with $10^{4}$ cells and normally distributed misorientation angles and terminated when 2000 cells remain. At this stage, the simulation is essentially stagnant. Five trials were executed and we consider the average of $\rho$ of the empirical GBCD's. Possible 'temperature' parameters $\lambda$ and $\rho_{\lambda}$ in (5.1) for the density (6.1) are constructed. This $\rho_{\lambda}$ then defines a trial relative entropy via (5.2). We now identify the parameter $\sigma$, which turns out to be $\sigma \approx 0.1$, and the value of the relative entropy $\Phi_{\sigma}\left(T_{\infty}\right) \approx 0.01$, which is about $10 \%$ of its initial value, Figure 6 . From Figure 7 (left), we see that this relative entropy $\Phi_{\sigma}$ has exponential decay until it reaches time about $t=1.5$, after which it remains constant. The averaged empirical GBCD is compared with the Boltzmann distribution in Figure 7 (right). The solution itself then tends exponentially in $L^{1}$ to its limit $\rho_{\sigma}$ by the Kullback-Leibler Inequality.
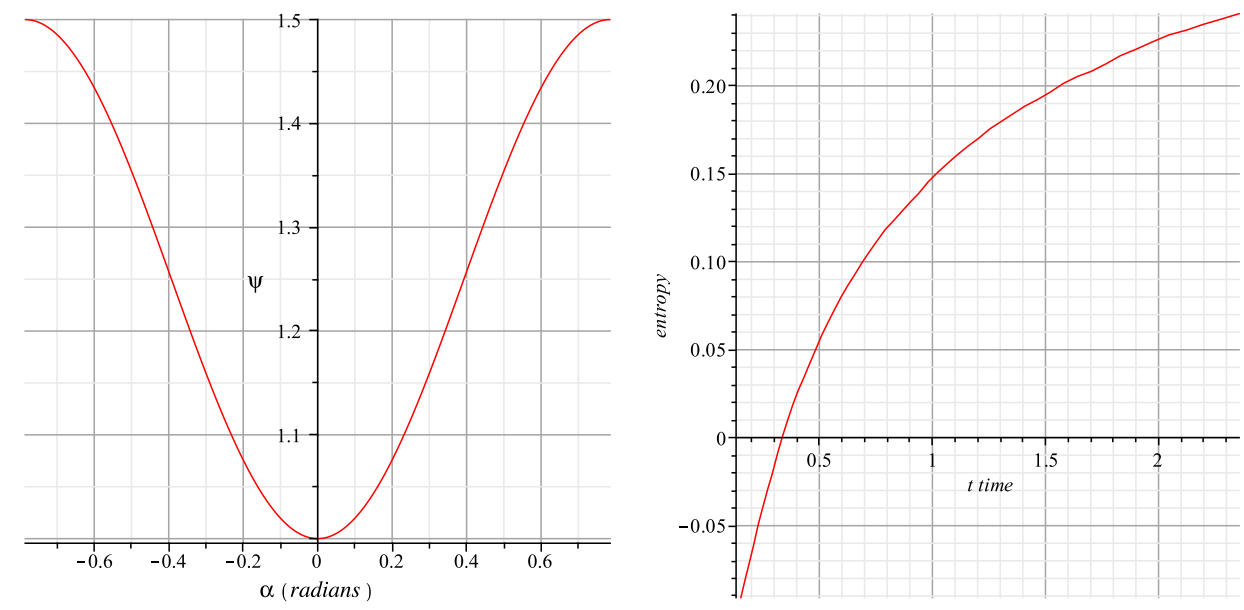

Figure 5. (left) The energy density $\psi(\alpha)=1+\epsilon \sin ^{2} 2 \alpha,|\alpha|<\pi / 4, \epsilon=\frac{1}{2}$. (right) The entropy of $\rho(\alpha, t)$ as a function of time $t$ is increasing, suggesting the development of order in the configuration.

6.2. Quartic interfacial energy density. Our second example is a quartic energy

$$
\psi(\alpha)=1+\epsilon(\sin 2 \alpha)^{4},-\frac{\pi}{4} \leqq \alpha \leqq \frac{\pi}{4}, \epsilon=1 / 2 .
$$



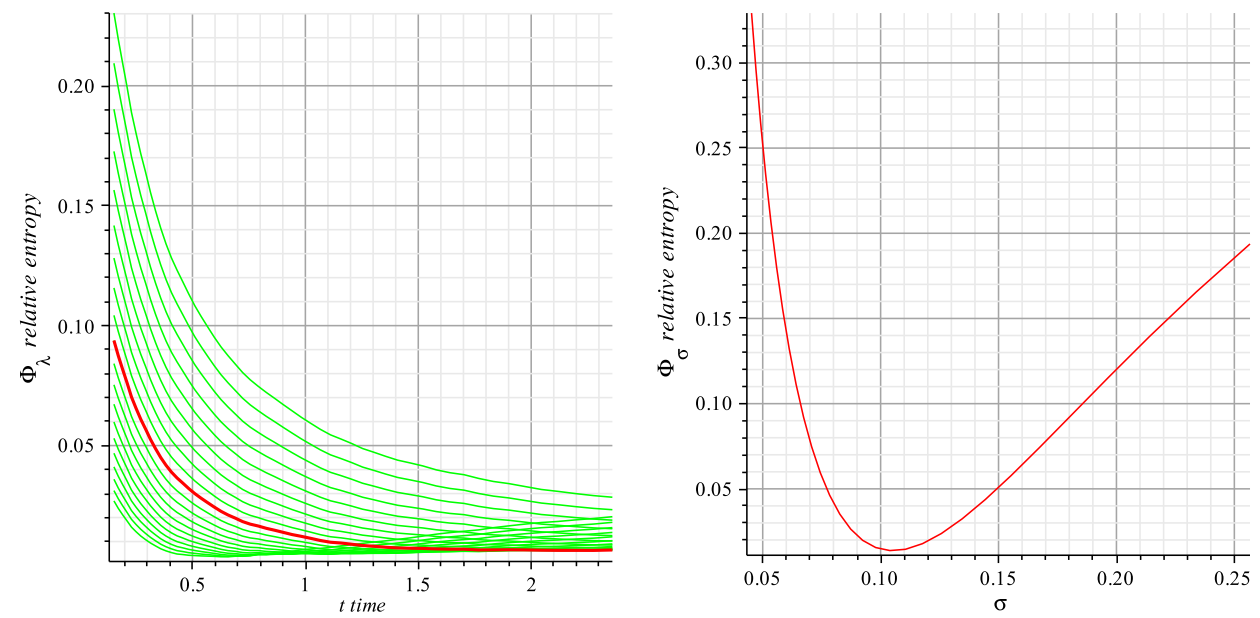

FIGURE 6. In these plots, the GBCD $\rho$ is averaged over 5 trials. (left) The relative entropy of the grain growth simulation with energy density (6.1) for a sequence of $\Phi_{\lambda}$ vs. $t$ with the optimal choice $\sigma \approx 0.1$ noted in red. (right) Relative entropy for an indicated range of values of temperature parameter $\lambda$ at the terminal time $t=2.3$. The minimum value of the relative entropy is $\approx 0.01$.
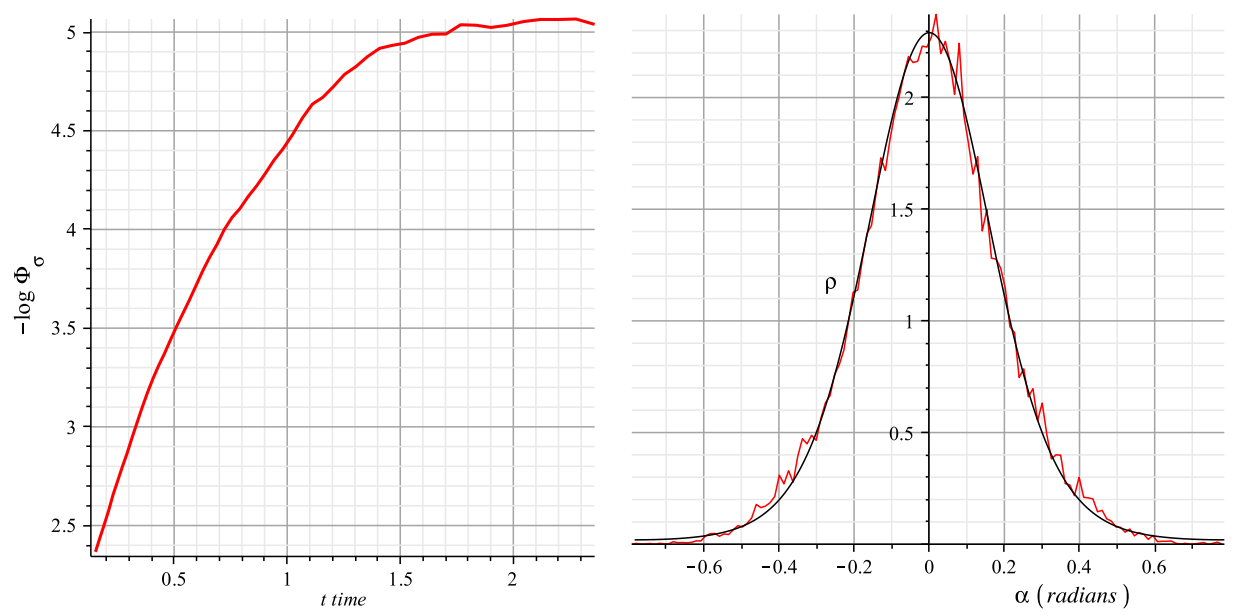

FigURE 7. In these plots, the GBCD is averaged over 5 trials. (left) Plot of $-\log \Phi_{\sigma}$ vs. $t$ with energy density (6.1). It is approximately linear until it becomes constant showing that $\Phi_{\sigma}$ decays exponentially.(right) GBCD $\rho$ (red) and Boltzmann distribution $\rho_{\sigma}$ (black) for the potential $\psi$ of (6.1) with parameter $\sigma \approx 0.1$ as predicted by our theory.

Again, a configuration of $10^{4}$ cells is initialized with normally distributed misorientations and, this time, the computation proceeds until about 1000 cells remain. The relative entropy and the equilibrium Boltzmann statistic stabilize when 2000 cells remain. Seven 


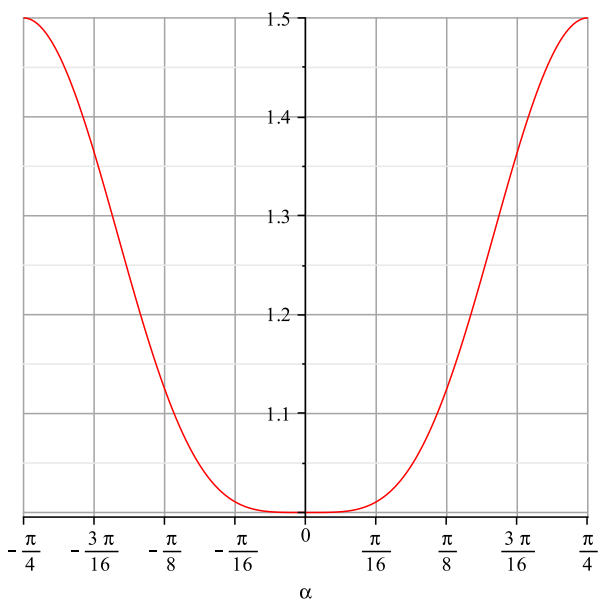

FigURE 8. The energy density $\psi(\alpha)=1+\epsilon \sin ^{4} 2 \alpha,|\alpha|<\pi / 4, \epsilon=\frac{1}{2}$.

trials were executed and we consider the average of $\rho$ of seven empirical GBCD's. Results are summarized in Fig. 9 and Fig. 10.
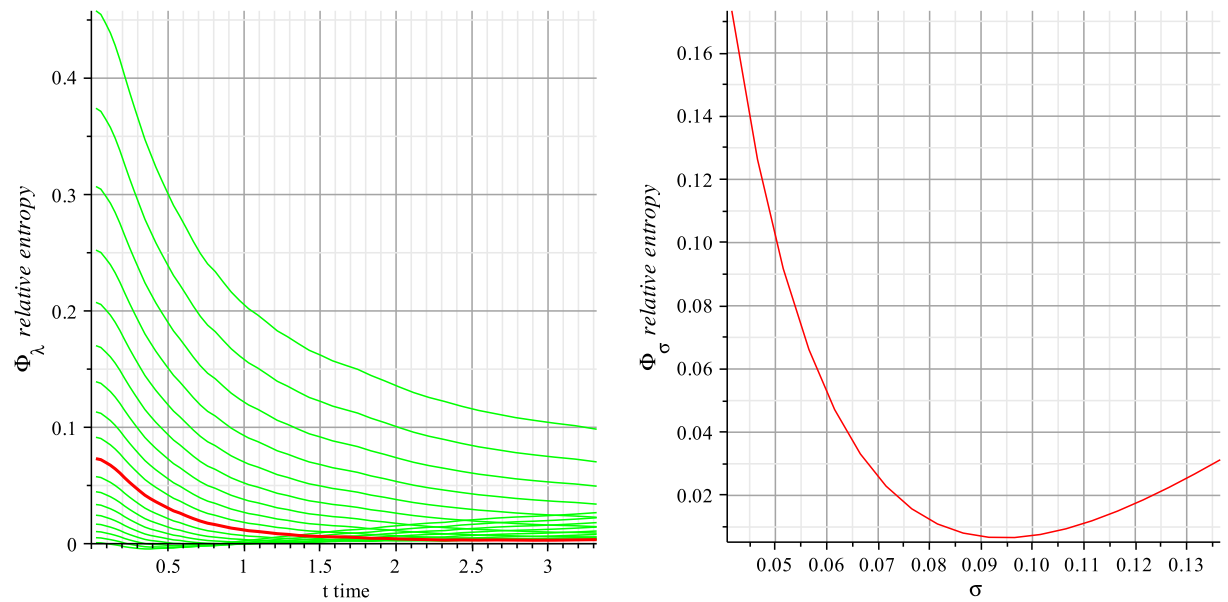

FiguRE 9. In these plots, the GBCD $\rho$ is averaged over 7 trials. (left) The relative entropy of the grain growth simulation with energy density (6.2) for a sequence of $\Phi_{\lambda}$ vs. $t$ with the optimal choice $\sigma \approx 0.095$ noted in red. (right) Relative entropy for an indicated range of values of temperature parameter $\sigma$ at the terminal time $\mathrm{t}=3$. The minimum value of the relative entropy is $\approx 0.007$. 


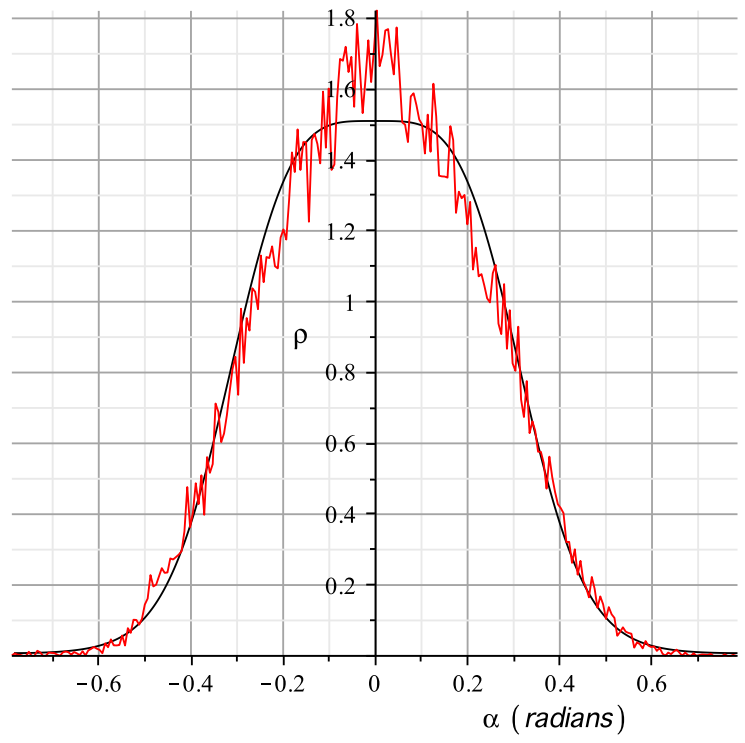

FIGURE 10. Comparison of the empirical distribution at time $T=3$, when $80 \%$ of the cells have been deleted, with $\rho_{\sigma}$, the Boltzmann distribution of (5.1), with $\sigma$ extracted from Fig.9. The GBCD $\rho$ is averaged over 7 trials.

6.3. Remarks on a Theory for the Diffusion Coefficient $\sigma$ or the TemperatureLike Parameter. The network level nonequlibrium nature of the iterative scheme introduced in our theory Sections 4 - 5, leaves free a temperature-like parameter $\sigma$. However, as we showed in Section 5, we can uniquely identify $\sigma$. But can we a priori determine or control this temperature-like parameter? There are different approaches to this question, none of which have been especially successful at this point. One possible approach is to consider a different theory that is developed for the simplified model based on the kinetic equations description in [16]. However, this particular description [16] would have to be improved, since it does not produce a very good result for $\sigma$ at this point. However, this method would still have only an empirical flavor: the value of $\sigma$ will be obtained once the solution of kinetic equations is computed. Another direction to consider here is based on the statistical analysis of the data obtained from many trials and to understand the possible connection to branching processes.

\section{Closing comments}

Engineering the microstructure of a material is a central task of materials science and its study gives rise to a broad range of basic science issues, as has been long recognized. Central to these issues is the coarsening of the cellular structure. Here we have outlined an entropy based theory of the GBCD which is an upscaling of cell growth according to the two most basic properties of a coarsening network: a local evolution law and space filling contraints. The theory accomodates the irreversibility conferred by the critical events or 
topological rearrangements which arise during coarsening. It adds to the body of evidence that the evolution of the boundary network is the primary origin of texture development. It accounts both for the GBCD and its kinetics.

There are many known environments where the kinetics of growth do not seem to follow this sort of pattern. Let us briefly consider one, stagnation in the evolution of metallic $(\mathrm{Cu}$ and $\mathrm{Al}$ ) thin films, important for the metallization of semiconductors, [12],[13]. Stagnation means that the growth process appears to stop even though the material remains in the furnace. Some progress is found in [36]. A striking feature of these films is a nearly exact log-normal distribution of the relative grain diameters based on a study of 27 samples consisting of 35,000 grains prepared in different experiments in a wide variety of conditions. The grain diameter is, basically, the square root of its area. This distribution is not found in any simulation of coarsening known to us. One possible starting point for an investigation is the well known Kolmogorov "rock crushing" problem, which has a representation as a scaled branching process.

The stagnation isssue is, of course, just a hint of the variety of challenges we encounter in this exciting field.

\section{ACKNOWLEDGEMENTS}

Much of this research was done while E. Eggeling, Y. Epshteyn and R. Sharp were postdoctoral associates at the Center for Nonlinear Analysis at Carnegie Mellon University. We are grateful to our colleagues G. Rohrer, A. D. Rollett, R. Schwab, and R. Suter for their collaboration.

\section{REFERENCES}

[1] B.L. Adams, D. Kinderlehrer, I. Livshits, D. Mason, W.W. Mullins, G.S. Rohrer, A.D. Rollett, D. Saylor, S Ta'asan, and C. Wu. Extracting grain boundary energy from triple junction measurement. Interface Science, 7:321-338, 1999.

[2] BL Adams, D Kinderlehrer, WW Mullins, AD Rollett, and S Ta'asan. Extracting the relative grain boundary free energy and mobility functions from the geometry of microstructures. Scripta Materiala, 38(4):531-536, Jan 131998.

[3] Luigi Ambrosio, Nicola Gigli, and Giuseppe Savaré. Gradient flows in metric spaces and in the space of probability measures. Lectures in Mathematics ETH Zürich. Birkhäuser Verlag, Basel, second edition, 2008.

[4] Todd Arbogast. Implementation of a locally conservative numerical subgrid upscaling scheme for twophase Darcy flow. Comput. Geosci., 6(3-4):453-481, 2002. Locally conservative numerical methods for flow in porous media.

[5] Todd Arbogast and Heather L. Lehr. Homogenization of a Darcy-Stokes system modeling vuggy porous media. Comput. Geosci., 10(3):291-302, 2006.

[6] Matthew Balhoff, Andro Mikelić, and Mary F. Wheeler. Polynomial filtration laws for low Reynolds number flows through porous media. Transp. Porous Media, 81(1):35-60, 2010.

[7] Matthew T. Balhoff, Sunil G. Thomas, and Mary F. Wheeler. Mortar coupling and upscaling of porescale models. Comput. Geosci., 12(1):15-27, 2008.

[8] K. Barmak. unpublished.

[9] K. Barmak, E. Eggeling, M. Emelianenko, Y. Epshteyn, D. Kinderlehrer, R.Sharp, and S.Ta'asan. Predictive theory for the grain boundary character distribution. In Materials Science Forum, volume 715-716, pages 279-285. Trans Tech Publications, 2012. 
[10] K. Barmak, E. Eggeling, M. Emelianenko, Y. Epshteyn, D. Kinderlehrer, R. Sharp, and S. Ta'asan. Critical events, entropy, and the grain boundary character distribution. Phys. Rev. B, 83(13):134117, Apr 2011.

[11] K. Barmak, E. Eggeling, M. Emelianenko, Y. Epshteyn, D. Kinderlehrer, and S. Ta'asan. Geometric growth and character development in large metastable systems. Rendiconti di Matematica, Serie VII, 29:65-81, 2009.

[12] K. Barmak, E. Eggeling, R. Sharp, S. Roberts, T. Shyu, T. Sun, B. Yao, S. Ta'asan, D. Kinderlehrer, A. Rollett, and K. Coffey. Grain growth and the puzzle of its stagnation in thin films: A detailed comparison of experiments and simulations. In Materials Science Forum, volume 715-716, pages 473479. Trans Tech Publications, 2012.

[13] K. Barmak, E. Eggeling, R. Sharp, S. Ta'asan, D. Kinderlehrer, A. Rollett, and K. Coffey. Grain growth and the puzzle of its stagnation in thin films: A detailed comparison of experiments and simulations. submitted, 2012.

[14] K. Barmak, M. Emelianenko, D. Golovaty, D. Kinderlehrer, and S. Ta'asan. On a statistical theory of critical events in microstructural evolution. In Proceedings CMDS 11, pages 185-194. ENSMP Press, 2007.

[15] K. Barmak, M. Emelianenko, D. Golovaty, D. Kinderlehrer, and S. Ta'asan. Towards a statistical theory of texture evolution in polycrystals. SIAM Journal Sci. Comp., 30(6):3150-3169, 2007.

[16] K. Barmak, M. Emelianenko, D. Golovaty, D. Kinderlehrer, and S. Ta'asan. A new perspective on texture evolution. International Journal on Numerical Analysis and Modeling, 5(Sp. Iss. SI):93-108, 2008.

[17] Katayun Barmak, Eva Eggeling, Maria Emelianenko, Yekaterina Epshteyn, David Kinderlehrer, Richard Sharp, and Shlomo Ta'asan. An entropy based theory of the grain boundary character distribution. Discrete Contin. Dyn. Syst., 30(2):427-454, 2011.

[18] Jean-David Benamou and Yann Brenier. A computational fluid mechanics solution to the MongeKantorovich mass transfer problem. Numer. Math., 84(3):375-393, 2000.

[19] G. Bertotti. Hysteresis in magnetism. Academic Press, 1998.

[20] Lia Bronsard and Fernando Reitich. On three-phase boundary motion and the singular limit of a vector-valued Ginzburg-Landau equation. Arch. Rational Mech. Anal., 124(4):355-379, 1993.

[21] J.E. Burke and D. Turnbull. Recrystallization and grain growth. Progress in Metal Physics, 3(C):220 244,IN11-IN12,245-266,IN13-IN14,267-274,IN15,275-292, 1952. cited By (since 1996) 68.

[22] Philippe G. Ciarlet. The finite element method for elliptic problems. North-Holland Publishing Co., Amsterdam, 1978. Studies in Mathematics and its Applications, Vol. 4.

[23] Antonio DeSimone, Robert V. Kohn, Stefan Müller, Felix Otto, and Rudolf Schäfer. Twodimensional modelling of soft ferromagnetic films. R. Soc. Lond. Proc. Ser. A Math. Phys. Eng. Sci., 457(2016):2983-2991, 2001.

[24] Matt Elsey, Selim Esedoglu, and Peter Smereka. Diffusion generated motion for grain growth in two and three dimensions. J. Comput. Phys., 228(21):8015-8033, 2009.

[25] Y. Epshteyn and B. Rivière. On the solution of incompressible two-phase flow by a p-version discontinuous Galerkin method. Comm. Numer. Methods Engrg., 22:741-751, 2006.

[26] Y. Epshteyn and B. Rivière. Fully implicit discontinuous finite element methods for two-phase flow. Applied Numerical Mathematics, 57:383-401, 2007.

[27] M Frechet. Sur la distance de deux lois de probabilite. Comptes Rendus de l'Academie des Sciences Serie I-Mathematique, 244(6):689-692, 1957.

[28] Harald Garcke, Britta Nestler, and Barbara Stoth. A multiphase field concept: numerical simulations of moving phase boundaries and multiple junctions. SIAM J. Appl. Math., 60(1):295-315 (electronic), 2000 .

[29] S. K. Godunov. A difference method for numerical calculation of discontinuous solutions of the equations of hydrodynamics. Mat. Sb. (N.S.), 47 (89):271-306, 1959. 
[30] S. K. Godunov and V. S. Ryaben'kii. Difference schemes, volume 19 of Studies in Mathematics and its Applications. North-Holland Publishing Co., Amsterdam, 1987. An introduction to the underlying theory, Translated from the Russian by E. M. Gelbard.

[31] Robert Gomer and Cyril Stanley Smith, editors. Structure and Properties of Solid Surfaces, Chicago, 1952. The University of Chicago Press. Proceedings of a conference arranged by the National Research Council and held in September, 1952, in Lake Geneva, Wisconsin, USA.

[32] M. Gurtin. Thermomechanics of evolving phase boundaries in the plane. Oxford, 1993.

[33] R. Helmig. Multiphase flow and transport processes in the subsurface. Springer, 1997.

[34] C. Herring. Surface tension as a motivation for sintering. In Walter E. Kingston, editor, The Physics of Powder Metallurgy, pages 143-179. Mcgraw-Hill, New York, 1951.

[35] C. Herring. The use of classical macroscopic concepts in surface energy problems. In Gomer and Smith [31], pages 5-81. Proceedings of a conference arranged by the National Research Council and held in September, 1952, in Lake Geneva, Wisconsin, USA.

[36] E. A. Holm and S. M. Foiles. Grain growth stagnation caused by the grain boundary roughening transition. In Materials Science Forum, volume 715-716, pages 415-415. Trans Tech Publications, 2012.

[37] Arieh Iserles. A first course in the numerical analysis of differential equations. Cambridge Texts in Applied Mathematics. Cambridge University Press, Cambridge, 1996.

[38] R Jordan, D Kinderlehrer, and F Otto. Free energy and the fokker-planck equation. Physica D, 107(24):265-271, Sep 11997.

[39] R Jordan, D Kinderlehrer, and F Otto. The variational formulation of the fokker-planck equation. SIAM J. Math. Analysis, 29(1):1-17, Jan 1998.

[40] S.G. Kim, D.I. Kim, W.T. Kim, and Y.B. Park. Computer simulations of two-dimension and threedimensional ideal grain growth. Phys. Rev. E, 74, 2006.

[41] D Kinderlehrer, J Lee, I Livshits, A Rollett, and S Ta'asan. Mesoscale simulation of grain growth. Recrystalliztion and grain growth, pts 1 and 2, 467-470(Part 1-2):1057-1062, 2004.

[42] D Kinderlehrer and C Liu. Evolution of grain boundaries. Mathematical Models and Methods in Applied Sciences, 11(4):713-729, Jun 2001.

[43] D Kinderlehrer, I Livshits, GS Rohrer, S Ta'asan, and P Yu. Mesoscale simulation of the evolution of the grain boundary character distribution. Recrystallization and grain growth, pts 1 and 2, 467-470(Part 1-2):1063-1068, 2004.

[44] David Kinderlehrer, Irene Livshits, and Shlomo Ta'asan. A variational approach to modeling and simulation of grain growth. SIAM J. Sci. Comp., 28(5):1694-1715, 2006.

[45] Robert V. Kohn. Irreversibility and the statistics of grain boundaries. Physics, 4:33, Apr 2011.

[46] Robert V. Kohn and Felix Otto. Upper bounds on coarsening rates. Comm. Math. Phys., 229(3):375395, 2002.

[47] L. D. Landau and E. M. Lifshitz. Fluid mechanics. Translated from the Russian by J. B. Sykes and W. H. Reid. Course of Theoretical Physics, Vol. 6. Pergamon Press, London, 1959.

[48] Peter D. Lax. Weak solutions of nonlinear hyperbolic equations and their numerical computation. Comm. Pure Appl. Math., 7:159-193, 1954.

[49] Peter D. Lax. Hyperbolic systems of conservation laws and the mathematical theory of shock waves. Society for Industrial and Applied Mathematics, Philadelphia, Pa., 1973. Conference Board of the Mathematical Sciences Regional Conference Series in Applied Mathematics, No. 11.

[50] Bo Li, John Lowengrub, Andreas Rätz, and Axel Voigt. Geometric evolution laws for thin crystalline films: modeling and numerics. Commun. Comput. Phys., 6(3):433-482, 2009.

[51] I.M. Lifshitz, E. M. and V.V. Slyozov. The kinetics of precipitation from suprsaturated solid solutions. Journal of Physics and Chemistry of Solids, 19(1-2):35-50, 1961.

[52] John S. Lowengrub, Andreas Rätz, and Axel Voigt. Phase-field modeling of the dynamics of multicomponent vesicles: spinodal decomposition, coarsening, budding, and fission. Phys. Rev. E (3), 79(3):0311926, 13, 2009. 
[53] W.W. Mullins. 2-Dimensional motion of idealized grain growth. Journal Applied Physics, 27(8):900904, 1956.

[54] W.W. Mullins. Solid Surface Morphologies Governed by Capillarity, pages 17-66. American Society for Metals, Metals Park, Ohio, 1963.

[55] W.W. Mullins. On idealized 2-dimensional grain growth. Scripta Metallurgica, 22(9):1441-1444, SEP 1988.

[56] Felix Otto, Tobias Rump, and Dejan Slepčev. Coarsening rates for a droplet model: rigorous upper bounds. SIAM J. Math. Anal., 38(2):503-529 (electronic), 2006.

[57] J. Rissanen. Complexity and information in data. In Entropy, Princeton Ser. Appl. Math., pages 299312. Princeton Univ. Press, Princeton, NJ, 2003.

[58] GS Rohrer. Influence of interface anisotropy on grain growth and coarsening. Annual Review of Materials Research, 35:99-126, 2005.

[59] Anthony D. Rollett, S.-B. Lee, R. Campman, and G. S. Rohrer. Three-dimensional characterization of microstructure by electron back-scatter diffraction. Annual Review of Materials Research, 37:627-658, 2007.

[60] Cyril Stanley Smith. Grain shapes and other metallurgical applications of topology. In Gomer and Smith [31], pages 65-108. Proceedings of a conference arranged by the National Research Council and held in September, 1952, in Lake Geneva, Wisconsin, USA.

[61] H. Bruce Stewart and Burton Wendroff. Two-phase flow: models and methods. J. Comput. Phys., 56(3):363-409, 1984.

[62] Andrea Toselli and Olof Widlund. Domain decomposition methods - algorithms and theory, volume 34 of Springer Series in Computational Mathematics. Springer-Verlag, Berlin, 2005.

[63] Cédric Villani. Topics in optimal transportation, volume 58 of Graduate Studies in Mathematics. American Mathematical Society, Providence, RI, 2003.

[64] J. Von Neumann and R. D. Richtmyer. A method for the numerical calculation of hydrodynamic shocks. J. Appl. Phys., 21:232-237, 1950.

[65] John von Neumann. Discussion remark concerning paper of C. S. Smith "grain shapes and other metallurgical applications of topology". In Gomer and Smith [31], pages 108-110. Proceedings of a conference arranged by the National Research Council and held in September, 1952, in Lake Geneva, Wisconsin, USA.

[66] C Wagner. Theorie der alterung von niederschlagen durch umlosen (Ostwald-Reifung). Zeitschrift fur Elektrochemie, 65(7-8):581-591, 1961.

E-mail address: katayun@andrew.cmu.edu

E-mail address: eva.eggeling@fraunhofer.at

E-mail address: memelian@gmu.edu

E-mail address: epshteyn@math.utah.edu

E-mail address: davidk@cmu.edu

E-mail address: sharp@andrew.cmu.edu

E-mail address: rsharp@gmail.com 